\title{
Electropolishing of nickel and cobalt in deep eutectic solvents
}

\author{
Wrya O. Karim ${ }^{\mathrm{a}, \mathrm{b}}$, Andrew P. Abbott, ${ }^{\text {a }}$ Salih Cihangir ${ }^{\mathrm{a}}$ and Karl S. Ryder ${ }^{\mathrm{a}}$ \\ ${ }^{\text {a }}$ Chemistry Department, University of Leicester, Leicester, UK, LE1 7RH. \\ ${ }^{\mathrm{b}}$ Chemistry Department, University of Sulaimani, Sulaimani, Iraq \\ E-mail: apa1@le.ac.uk;
}

\begin{abstract}
Electropolishing is a common method for decreasing surface roughness and removing surface irregularities. In this paper the electropolishing of nickel and cobalt are successfully demonstrated in a deep eutectic solvent, comprising a 2:1 molar mixture of ethylene glycol (EG) and choline chloride. Voltammetric and electrochemical impedance studies were used to characterise the polishing mechanism and show that film formation occurs prior to polishing. Scanning electron microscopy (SEM) and atomic force microscopy (AFM) were used to characterise the morphology before and after polishing and 3D optical microscopy was used in-situ to observe film formation during polishing. This study shows that the impact of film formation and subsequently mass transport factor are responsible for electropolishing of both metals in the choline chloride-based ionic liquid.
\end{abstract}

\section{Keywords}

Deep eutectic solvent, electropolishing, nickel, cobalt, film formation. 


\section{Introduction}

Electropolishing is essentially the controlled electrochemical corrosion of a metal to bring about a decrease in surface roughness. Electropolishing an item can increase the corrosion resistance of the component as it enables better, more homogeneous passivation and it can also decrease wear in moving systems. It has most commonly been studied in aqueous solutions primarily using concentrated mineral acids such as sulfuric, hydrochloric and phosphoric acids. The large scale usage of electropolishing is most frequently applied to stainless steel ${ }^{1}$ although other metals such as nickel, copper and titanium are also electropolished. ${ }^{2,3,4}$ The fundamental and practical aspects of electropolishing have been covered in two reviews. ${ }^{5,6}$ It is known that for electropolishing to occur, a viscous film generally forms on the metal surface. ${ }^{5}$ It is therefore essential to choose an electrolyte that will enable film formation on the metal surface. ${ }^{6}$ This process is not free from some practical issues, such as the corrosivity of the electrolyte, gas evolution and low current efficiency.

Recently, we have shown that deep eutectic solvents, DESs, can be applied to electropolishing. ${ }^{7}$ DESs are mixtures of quaternary ammonium salts and hydrogen bond donors such as urea and ethylene glycol. ${ }^{7}$ These were applied to the electropolishing of 316 stainless steel and it was shown that similar surface roughnesses could be obtained with similar voltages to those used in aqueous electropolishing. ${ }^{8}$ The study showed that a film formed on the electrode surface and this controlled the dissolution rate of the metal. It was also shown that during the polishing process no dealloying occurred showing that none of the metals were preferentially dissolved. ${ }^{9}$ The concept was later extended to the electropolishing of nickel superalloys using the same eutectic mixture. In this study it was shown that the etch could be made selective to either the $\gamma$ or $\gamma^{\prime}$ phase or could be made to etch both phases at the same rate depending on the applied potential. $^{10}$

In the present study, the electropolishing of pure nickel and cobalt were studied in a choline chloride based deep eutectic solvent using electrochemical, AC impedance and microscopic techniques to investigate the mechanism of dissolution and determine whether the metals could be electropolished. While a limited number of studies have investigated pure nickel in aqueous solutions no studies have been published on cobalt, although the electropolishing of a cobaltchromium alloy has been patented. ${ }^{11}$ It is shown that film formation on the metal surface is important and the mass transportation during the process is the key factor of achieving a polished surface finish which is evidenced by a.c. impedance results. 


\section{Experimental}

The DES was prepared by mixing choline chloride (ChCl) (Aldrich, 99\%) and ethylene glycol, (EG) (Aldrich, > 99\%) in a molar ratio of 1: 2 (1 ChCl: $2 \mathrm{EG})$. The mixture was heated at 60 ${ }^{\circ} \mathrm{C}$ with continuous stirring until a clear liquid formed.

The cyclic voltammetry and the AC impedance were performed using an Autolab PGSTAT 12 which was controlled by GPES software and fitted with an FRA impedance module. The collection of impedance spectra was in the frequency range of 1 to $65000 \mathrm{~Hz}$ with an amplitude of $10 \mathrm{mV}$ of the a.c. signal. All electrochemistry measurements were conducted in a three electrode cell involving $1 \mathrm{~mm}$ diameter metal disc ( $\mathrm{Ni}$ or $\mathrm{Co}$ ) working electrode, sealed in glass, a platinum flag $\left(1 \mathrm{~cm}^{2}\right.$ area $)$ as a counter electrode and $\mathrm{Ag} / \mathrm{AgCl}(0.1 \mathrm{M}$ in $1 \mathrm{ChCl}: 2$ EG) reference electrode. The voltammetric measurements are carried out at $20^{\circ} \mathrm{C}$ at $5 \mathrm{mVs}^{-1}$ sweep rate.

The microscopic studies were performed using scanning electron microscopy (SEM) (Philips XL30 ESEM) and the AFM image acquisition were recorded using a Digital Instruments Nanoscope 4 run using contact mode. A Zeta-20 3D Optical Profiler was used to image the polishing of the nickel surface during the voltammetric experiments.

\section{Results and discussion}

\section{Cyclic voltammetry}

Fig. $1 \boldsymbol{a}$ shows a cyclic voltammogram of a pure nickel disc electrode (1 mm diameter) in 1 $\mathrm{ChCl}: 2 \mathrm{EG}$ at $20{ }^{\circ} \mathrm{C}$. The potential sweep starts from $0.0 \mathrm{~V}$ and an oxidation process is observed on the positive sweep with a peak which is centred at $+0.5 \mathrm{~V}$. On the negative sweep from $+1.5 \mathrm{~V}$ a hysteresis loop is observed between 0.3 and $0.0 \mathrm{~V}$ which is generally characteristic of a change in the surface area of the electrode. A peak in the cathodic current is observed at about $-1.1 \mathrm{~V}$ which is characteristic of Ni electrodeposition. A very similar shaped voltammogram is observed for the electrolysis of a solution of $\mathrm{NiCl}_{2}$ in the same DES. ${ }^{12}$ Fig. $\boldsymbol{I b}$ shows the corresponding cyclic voltammetric response of pure cobalt ( $1 \mathrm{~mm}$ diameter) in 1 $\mathrm{ChCl}: 2 \mathrm{EG}$ at $20^{\circ} \mathrm{C}$. A very similar shaped voltammogram is obtained with the oxidation peak and on-set voltage for reduction occurring at about the same potentials. This again is very similar to the response obtained for the electrodeposition of cobalt from the same DES. ${ }^{13}$ 

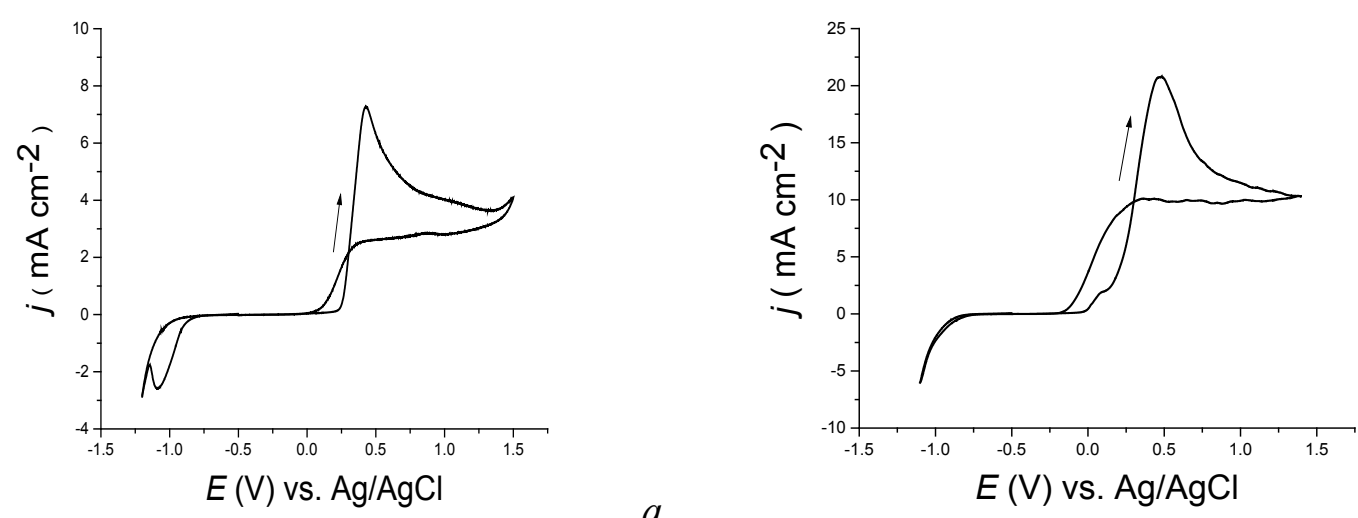

$a$

$b$

Fig. 1 Cyclic voltammograms of nickel (a) and cobalt (b) disc electrodes in $1 \mathrm{ChCl}: 2 \mathrm{EG}$ at a sweep rate $5 \mathrm{mVs}^{-1}$ at $20^{\circ} \mathrm{C}$.

The current density of the dissolution of Co is significantly larger (2.5 times) than that for $\mathrm{Ni}$. A similar electrochemical experiment was carried out with the same electrolyte using a 316 stainless steels (Cr 16, Ni, 10, Mo $2 \mathrm{wt} \%) .{ }^{1}$ It was found that the onset potential for anodic dissolution was $>1 \mathrm{~V}$, however if the electrode was abraded in situ the onset potential shifted to $0.3 \mathrm{~V}$ which is similar to that for Ni in Fig 1a. This suggested that the presence of an oxide on the surface was responsible for the large positive over-potential required to dissolve the stainless steel electrode.

\section{AC impedance spectra}

Fig. 2 shows AC impedance spectra of the nickel and cobalt electrodes in $1 \mathrm{ChCl}: 2 \mathrm{EG}$ at 20 ${ }^{\circ} \mathrm{C}$. The Nyquist plots for Co and Ni are very similar with characteristic Randle's circuits with Warburg elements. For Ni an electron transfer process is observed when the DC voltage is set at $+0.2 \mathrm{~V}$ which correlates with the onset voltage for the voltammogram. At $+0.4 \mathrm{~V}$ a clear Warburg response is observed corresponding to a diffusion controlled process. This could either be the diffusion of chloride to the electrode surface or diffusion of a metal based species from the electrode. Increasing the applied DC voltage has the effect of increasing the size of the semi-circle which results from a more resistive interfacial region and implies the formation of a film at the electrode surface.

Metal salts are known to be soluble in DESs. Previous work has characterised the speciation of metal salts in a selection of DESs and ionic liquids with discrete anions. It was found that many monovalent transition metal salts, e.g. $\mathrm{AgCl}$, formed complexes of the form $\left[\mathrm{MX}_{2}\right]^{-}$ whereas most divalent metals formed complexes of the form $\left[\mathrm{MX}_{4}\right]^{2-} \cdot{ }^{14}$ As one notable 
exception, nickel was observed to form complexes with the hydrogen bond donor of the DESs, instead of the anticipated chloro-complexes, as was observed for the corresponding cobalt salt. ${ }^{19}$

The electrodissolution of metals has previously been studied in the same DES to understand why dissolution was slow and it was found that copper clearly forms a green film on the electrode when anodically polarised as the interfacial region close to the electrode surface rapidly becomes saturated with metal ions and a neutral $\mathrm{CuCl}_{2}$ forms which precipitates on the electrode surface. ${ }^{15}$
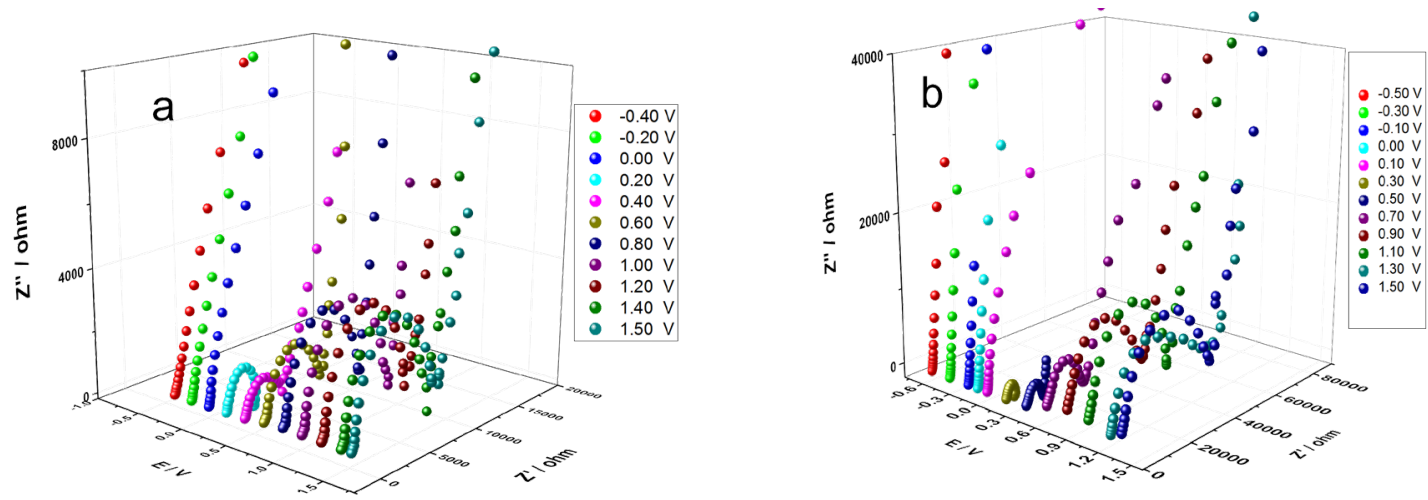

$a$

Fig. 2 Electrochemical impedance spectra of nickel (a) and cobalt (b) at various potentials with a.c. amplitude of $10 \mathrm{mV}$ in the frequency range of $1-65000 \mathrm{~Hz}$ at $20^{\circ} \mathrm{C}$.

$\mathrm{CV}$ and a.c. impedance spectroscopy of cobalt in $1 \mathrm{ChCl}: 2 \mathrm{EG}$ produces very similar to those observed for nickel. It can also be seen that the magnitude of the impedance semi-circle is about three times larger for cobalt than for nickel which would arise from a thicker or less conductive (less porous) film forming on the electrode surface. For the sake of comparison, if the films are assumed to both be homogeneous dielectrics then the thickness, $d$, can be estimated from the capacitance, $C$, of the film. Using;

$d=\varepsilon \varepsilon_{0} / C$

Where $\varepsilon$ is the static dielectric constant of the film material and $\varepsilon_{0}$ is the permittivity of free space. Assuming that the layer formed on the electrode surface is a metal chloride then the relative thicknesses of the films can be determined. The dielectric constant of $\mathrm{CoCl}_{2}$ is not known but given that most similar transition metal halides are in the range 5.3 to 11.2 , a value of 8.0 will be assumed. ${ }^{16}$ Using this value, the film forming on the nickel and cobalt surfaces can be calculated to be 0.98 and $14 \mu \mathrm{m}$, respectively. This suggests that the film formed on 
cobalt electrode surfaces is thicker than that formed on nickel and the a.c. impedance result data confirm that. $\mathrm{CV}$ and a.c. impedance spectroscopy of cobalt in $1 \mathrm{ChCl}: 2 \mathrm{EG}$ produces very different responses to those observed in the previous study for copper. It can be seen that the oxidative current decreases less abruptly than was the case with copper. It can also be seen that the magnitude of the impedance semi-circle is significantly larger for cobalt than for copper. $^{15}$

Fig. 3 shows a series of 3DM images of a nickel surface at various potentials on the forward (F) and backward scan (B) of a cyclic voltammogram in $1 \mathrm{ChCl}: 2 \mathrm{EG}$ at a sweep rate $5 \mathrm{mVs}^{-}$ 1. In the initial image at $0 \mathrm{~V}$, a variety of surface scratches can be observed on the metallic surface. In the time taken to proceed to $0.45 \mathrm{~V}(90 \mathrm{~s})$ the surface of the electrode has changed to a darker grey and a few of the surface features have been lost. At $0.6 \mathrm{~V}(120 \mathrm{~s})$ the surface scratches have largely disappeared and some regions have started to brighten while others have become darker. This trend continues through $0.8 \mathrm{~V}$ up to $1.5 \mathrm{~V}$ where bright metallic features become apparent. It could be concluded that the dark film that forms on the electrode surface between 0.45 and $0.8 \mathrm{~V}$ is responsible for limiting the diffusion of species to and from the metal surface.
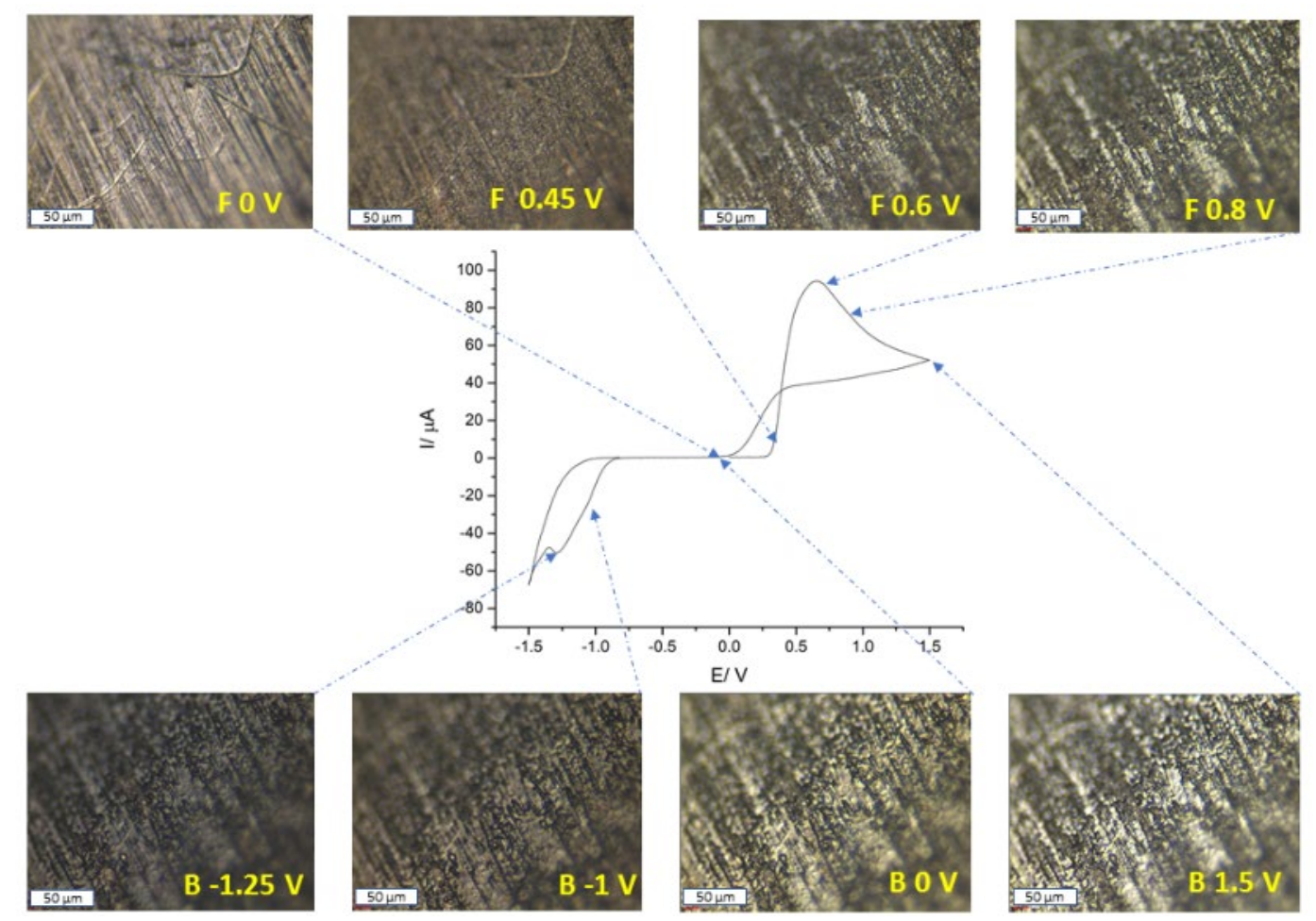

Fig. 3: 3DM images of a nickel surface at various potentials on the forward $(F)$ and backward scan (B) of a cyclic voltammogram in $1 \mathrm{ChCl} 2 \mathrm{EG}$ at a sweep rate $5 \mathrm{mVs}^{-1}$ at $20{ }^{\circ} \mathrm{C}$. 
On the back scan the bright surface start to darken slightly as the potential approaches $0 \mathrm{~V}$ but the surface features do not change significantly i.e. negligible bulk material has been removed in approximately $3 \mathrm{~min}$ so no obvious polishing occurs. At potentials below $-1 \mathrm{~V}$ the surface darkens as nickel is redeposited on the electrode surface.

\section{Surface morphology}

Electropolishing of nickel was carried out in $1 \mathrm{ChCl}$ : $2 \mathrm{EG}$ using a galvanostatic technique. It was found that at low current densities $<10 \mathrm{~mA} \mathrm{~cm}{ }^{-2}$, a dull surface finished was obtained on the nickel surface while significant hydrogen gas evolution occurred on the cathode at current densities $>50 \mathrm{~mA} \mathrm{~cm}^{-2}$. The hydrogen must originate from either ethylene glycol or trace of water. It was found that the optimum applied current densities were in the range (10-50 mA $\mathrm{cm}^{-2}$ ) in which a mirror finished surface for nickel could be obtained. This is close to the optimum range found for stainless steel which was shown to be about $50 \mathrm{~mA} \mathrm{~cm}{ }^{-2}{ }^{1}$ Fig. 4 shows the surface finish obtained at $12 \mathrm{~mA} \mathrm{~cm}^{-2}$, for $30 \mathrm{~min}$. the surface finish is similar to that obtained with stainless steel. ${ }^{1,8}$

The SEM image is shown in Fig. $\mathbf{4} \boldsymbol{b}$ where the unpolished and polished region are clearly visible which is separated by a boundary (white band). The polished region of nickel sample looks very smooth with no trace whereas grain boundaries are seen in the unpolished region. The surface morphology before and after electropolishing were studied using atomic force microscopy and the results are shown in Fig. $4 \boldsymbol{c}-f$. The nickel sheet prior to polishing was relatively rough with a lot of micro-roughness displaying sharp features in the $\mathrm{z}$ direction across the whole sample. The polished surface in Fig. $4 \boldsymbol{d}$ shows micro-roughness of less than $5 \mathrm{~nm}$ although the macro-roughness is still similar to that in the unpolished surface.

This shows the process is brightening which allows the surface to passivate. The corresponding SEM image shows how microscopic roughness is fully removed in the electropolishing process. It is interesting to notice that the success of electropolishing of nickel in $1 \mathrm{ChCl}: 2 \mathrm{EG}$ is similar to that observed previously for 316 stainless steel. ${ }^{8}$ 
a)

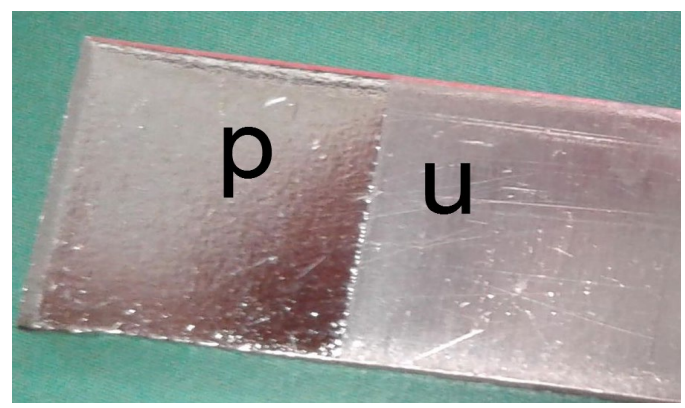

c) $\boldsymbol{R} \boldsymbol{a}=68 \mathrm{~nm}$

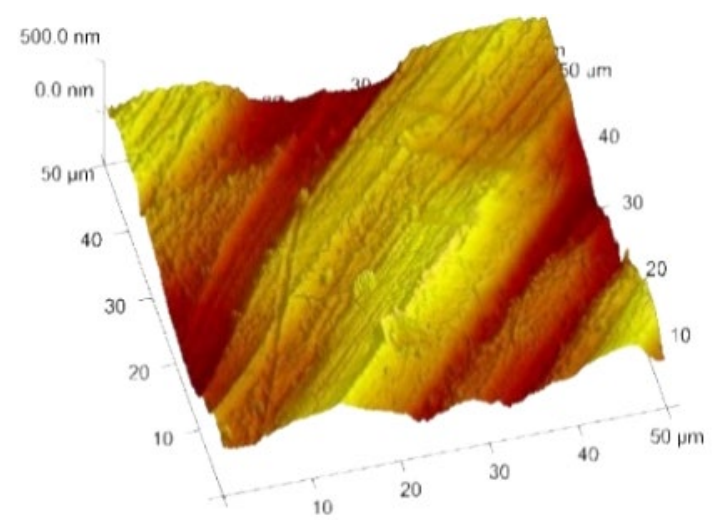

e)

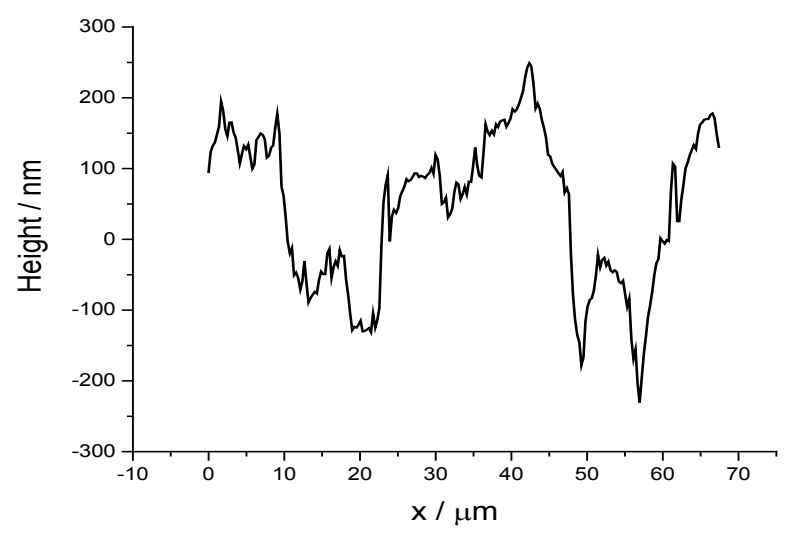

b)

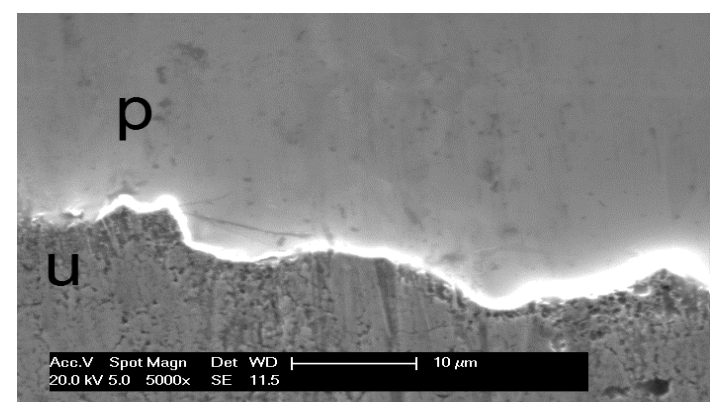

d) $\boldsymbol{R} \boldsymbol{a}=42 \mathrm{~nm}$

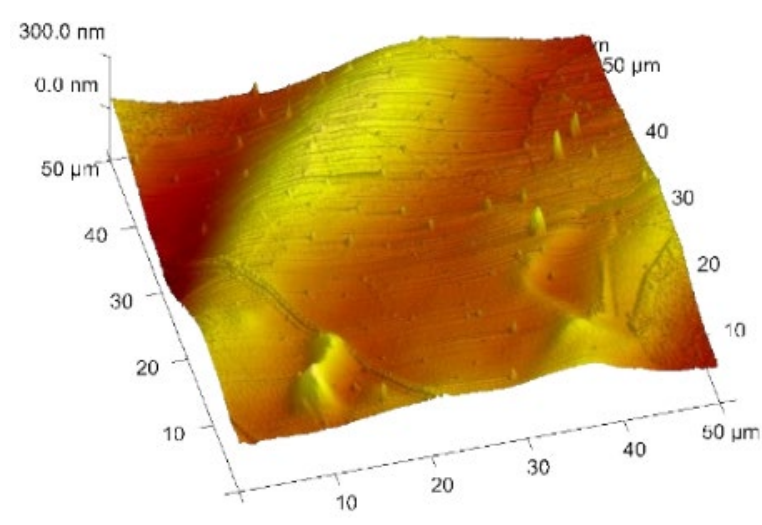

f)

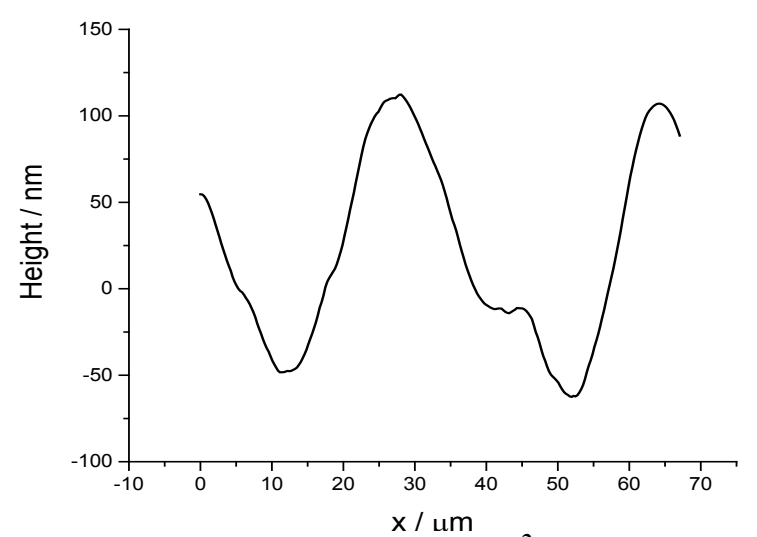

Fig. 4 a) Photograph of a sample of nickel sheet after dissolution $\left(12 \mathrm{~mA} \mathrm{~cm} \mathrm{~cm}^{-2}\right.$, for $\left.30 \mathrm{~min}\right)$ in $1 \mathrm{ChCl}$ : 2 EG at $20^{\circ} \mathrm{C}$, unpolished (u) and polished (p).b) SEM image of the interface between the polished and unpolished regions. c-f) AFM images, with single line traces alongside, of nickel recorded in resonant mode at a frequency of (ca. $300 \mathrm{kHz}$ ) (recorded in air at a scan rate of $0.5 \mathrm{~Hz}, 256$ lines) of the nickel sheet, c) and e) unpolished, d) and f) polished.

The same electropolishing process was carried out using cobalt as the electrode material. It was found that the range of current densities that could be applied to obtain an electropolished surface was in the range $35 \mathrm{~mA} \mathrm{~cm}^{-2}$ to $60 \mathrm{~mA} \mathrm{~cm}^{-2}$. The current density is slightly higher than that required for nickel due most probably to the thicker oxide film formed on the electrode surface. Fig. $5 \boldsymbol{a}$ shows a photograph of the surface finish obtained for cobalt in $1 \mathrm{ChCl}$ : $2 \mathrm{EG}$ 
after galvanostatic dissolution at $40 \mathrm{~mA} \mathrm{~cm}^{-2}$, for $40 \mathrm{~min}$ at $20^{\circ} \mathrm{C}$. It is interesting to note that electropolishing of nickel, cobalt and stainless steel all occur at over a limited current density range which is roughly the same for all three metals. This suggests that the kinetics of polishing is critical which ties in with the impedance data which shows that diffusion is critical for the polishing process.
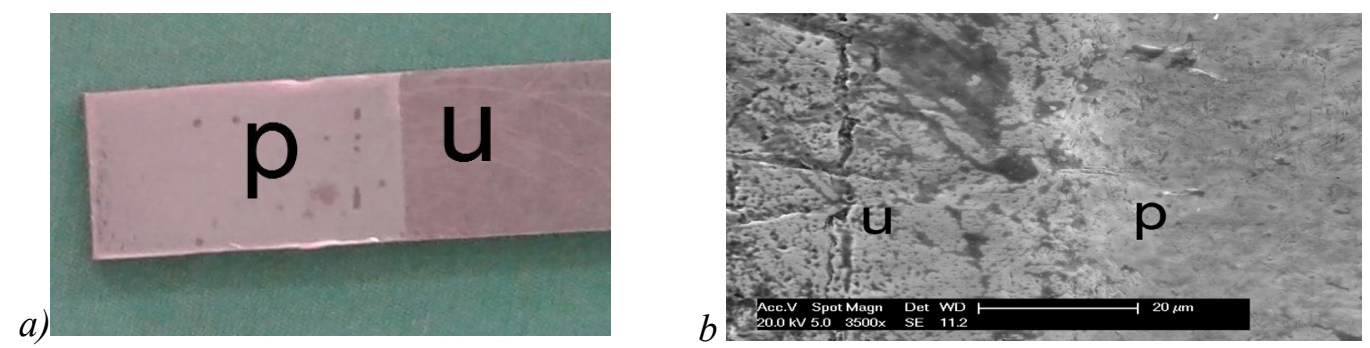

c) $R a=71 \mathrm{~nm}$

d) $R a=38 \mathrm{~nm}$
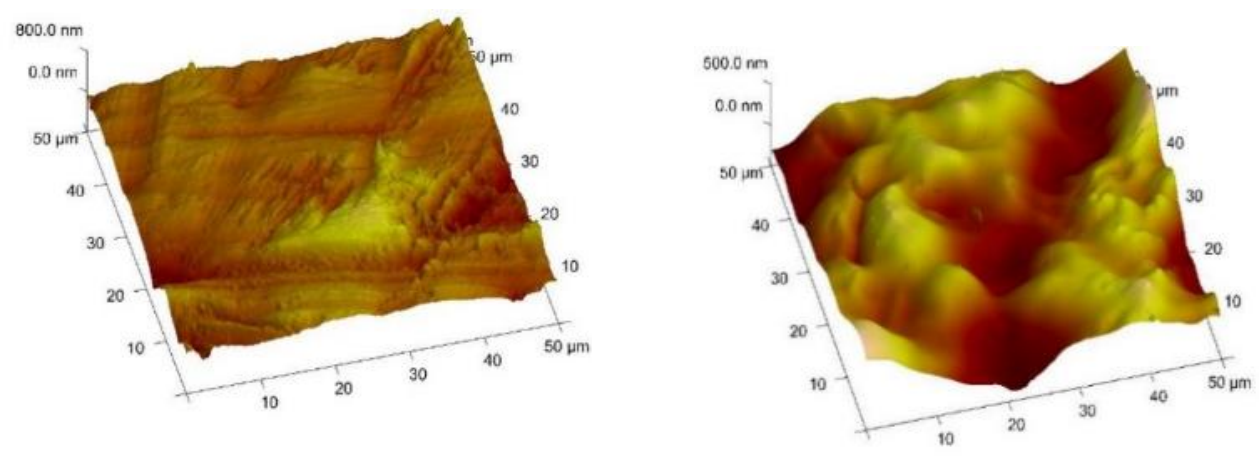

e)

f)
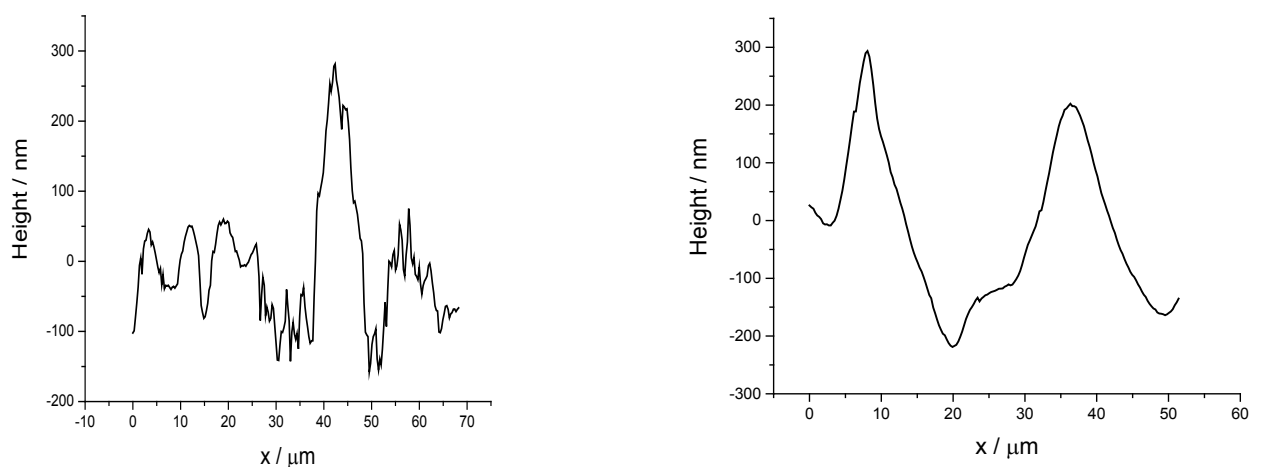

Fig. 5a Photograph of a sample of cobalt sheet after dissolution in $1 \mathrm{ChCl}: 2 \mathrm{EG}$ at $30 \mathrm{~mA}$ $\mathrm{cm}^{-2}$ for 30 min at $20^{\circ} \mathrm{C}$. b SEM image of the interface between the polished and unpolished regions $\boldsymbol{c}-f$ AFM images, with single line traces alongside, of cobalt recorded in resonant mode at a frequency of (ca. $300 \mathrm{kHz}$ ) (recorded in air at a scan rate of $0.5 \mathrm{~Hz}, 256$ lines) c) and e) unpolished, d) and f) polished. 
Fig. $5 \boldsymbol{b}$ shows the SEM image of the interface between the polished and unpolished region and just as in the case with nickel it can be seen that the scratches and machine marks are fully removed in the electropolishing process.

Using a current density of $30 \mathrm{~mA} \mathrm{~cm}^{-2}$ assuming the Co is oxidised with $100 \%$ current efficiency and goes to a $\mathrm{Co}^{\mathrm{II}}$ species the number of moles oxidised per minute equates to 1.86 x $10^{-5} \mathrm{~mol} \mathrm{~min}^{-1} \mathrm{~cm}^{-2}$. This corresponds to a mass loss of $1.09 \times 10^{-3} \mathrm{~g} \mathrm{~min}^{-1} \mathrm{~cm}^{-2}$. Using a density of $8.9 \mathrm{~g} \mathrm{~cm}^{-3}$ for Co the thickness of Co lost is approximately $1.12 \mu \mathrm{m} \mathrm{min}^{-1}$. For a 30 minute polish this should equate to a loss of $37 \mu \mathrm{m}$. 3DM of the step between the polished and unpolished region showed that the average step was approximately $26 \mu \mathrm{m}$. This is clearly less than expected but this is mostly due to the assumption about the current efficiency of the process. The same analysis for nickel was performed at $20 \mathrm{~mA} \mathrm{~cm}^{-2}$ for 18 mins with giving $14.8 \mu \mathrm{m}$ which is not what it was obtained from 3DM $(6 \mu \mathrm{m})$ as is presented in $\boldsymbol{F i g} . \mathbf{4 b}$.

The surface morphology of cobalt before and after electropolishing was also studied using atomic force microscopy and the results are shown in Fig. 5. The roughness of the cobalt sheet prior to polishing was similar to that for nickel in Fig. $4 c$. The polished surface shows a microroughness of less than $5 \mathrm{~nm}$ although the macro-roughness is still similar to that in the unpolished surface. This shows the brightening process is almost identical to that observed above for nickel and previously for stainless steel. ${ }^{1}$

\section{Conclusions}

This study has shown that cobalt and nickel can be electropolished successfully in $1 \mathrm{ChCl}: 2$ $\mathrm{EG}$ at $20^{\circ} \mathrm{C}$. Electrochemical impedance spectroscopy (EIS) has shown that the mechanism of electrodissolution for these two metals where a film forms on the electrode surface and a diffusional Warburg impedance is seen in the systems which electropolish. This confirms that the kinetics of polishing is critical which ties in with the impedance data which shows that mass transportation through the film formed, i.e. the diffusion of solvated oxidised metals through the film, is critical for the polishing process. This behaviour is almost identical to that observed for stainless steel in the same DES. Electropolishing of these three metals results in a very low micro-roughness of the surface.

\section{Acknowledgements}

The authors would like to thank the Human Capacity Development Program from the Kurdistan Government (WK) and Turkish Ministry of Education (SC) for scholarships. Some of the images for this study are taken from the PhD thesis of Dr W. Karim. ${ }^{17}$ 


\section{References}

${ }^{1}$ A. P. Abbott, G. Capper, K. J. McKenzie, A. Glidle and K. S. Ryder, Phys. Chem. Chem. Phys., 2006, 8, 4214-4221.

2 I. L. Alanis and D. J. Schiffrin, Electrochimica Acta, 1982, 27, 837-845.

${ }^{3}$ O. Piotrowski, C. Madore and D. Landolt, Journal of the Electrochemical Society, 1998, 145, 2362-2369.

${ }^{4}$ B. Du and I. I. Suni, Journal of The Electrochemical Society, 2004, 151, C375-C378.

${ }^{5}$ D. Landolt, Electrochimica Acta, 1987, 32, 1-11.

${ }^{6}$ S. Mohan, D. Kanagaraj, R. Sindhuja, S. Vijayalakshmi and N. Renganathan, Trans. Inst. Met. Finish., 2001, 79, 140-142.

${ }^{7}$ E. L. Smith, A. P. Abbott and K. S. Ryder, Chem. Rev., 2014, 114, 11060-82.

${ }^{8}$ A. P. Abbott, G. Capper, B. G. Swain and D. A. Wheeler, Trans. Inst. Met. Finish., 2005, 83, $51-53$.

${ }^{9}$ A. P. Abbott, G. Capper, K. J. McKenzie and K. S. Ryder, Electrochim. Acta, 2006, 51, 44204425 .

${ }^{10}$ A. P. Abbott, N. Dsouza, P. Withey and K. S. Ryder, Trans. Inst. Met. Finish., 2012, 90, 914.

${ }^{11}$ A. S. Andreacchi, US patent 7357854 B1, 2008.

${ }^{12}$ A. P. Abbott, A. Ballantyne, R. C. Harris, J. A. Juma and K. S. Ryder, Electrochim. Acta, 2015, $176718-726$.

${ }^{13}$ J. Juma, PhD thesis, University of Leicester, 2017.

${ }^{14}$ J. M. Hartley, C.-M. Ip, G. C. H. Forrest, K. Singh, S. J. Gurman, K. S. Ryder, A. P. Abbott and G. Frisch, Inorg. Chem., 2014, 53, 6280-6288.

${ }^{15}$ A. P. Abbott, G. Frisch J. M. Hartley, W. O. Karim and K. S. Ryder, Prog. Nat. Sci. Mat. $2015,25,595-602$.

${ }^{16}$ W. M. Haynes and D. R. Lide, CRC Handbook of Chemistry and Physics, CRC press, 2010.

${ }^{17}$ W. Karim, PhD thesis, University of Leicester, 2016. 